\title{
Review of: "Nicotine pouches- a research and regulatory policy agenda to maximise public health benefits and minimise harms"
}

\author{
Andrzej Sobczak
}

Potential competing interests: In 2015, AS accepted personal honoraria from the eSmoking Institute in Poznań, Poland, and nonfinancial support from Chic Group, Ltd., a manufacturer of electronic cigarettes in Poland.

This article provides an overview of existing nicotine pouches research that has been published in peer-reviewed journals. Nicotine pouches appeared on the market at the beginning of this century under the Zonnic brand, but they did not gain much popularity. Now Zonnic pouches are only available in the Scandinavian countries. The current versions appeared on the market a few years ago. According to the information contained in this study, they are available in five countries and in the EU. The authors list 11 producers, but the list is probably not complete. For example, nicotine pouches of the Polish producer 77 Group Ltd (Brand: 77; Country: Poland; Nicotine level: 3,6,12,20 mg) have been available in Poland for over a year. [1]

The authors of the reviewed work identified 7 papers on nicotine pouches, two of them are a letter to the editor and the industry watch. Although the number of analyzed works is small, this article is valuable for two reasons.

First, it is very likely that nicotine pouches are the least harmful among alternative tobacco products on the market that deliver nicotine (e-cigarettes, snus, tobacco heated product). Therefore, they can play a large role in reducing the harm caused by smoking.

Second, the authors highlighted research gaps in assessing their harmfulness and potential benefits, and possible regulatory approaches to ensure that these products do not lead to unintended consequences.

Referring to the item "Product chemistry". I believe that only nicotine is responsible for the toxicity of nicotine pouches. There is no indication that flavorings (as long as those used in the food industry are added) will have "potential toxic effects" as they enter the body through the digestive system. The toxicity of flavorings is a frequently raised argument in relation to e-cigarettes. However, the fundamental difference between the two products should be emphasized. Generation of aerosol in e-cigarettes occurs at temperatures above $200 \mathrm{C}$, which may result in flavorings degradation to produce potentially toxic compounds. Aromatics and their degradation products enter the body through the respiratory system and not through the digestive system. This can completely change their toxicity. However, according to the report of The Scientific Committee on Health, Environmental and Emerging Risks (SCHEER), to date there are no specific data showing that certain flavorings used in the EU pose a health risk to e-cigarette users following repeated exposure [2]. The only danger of using nicotine pouches I see is the possibility of causing local irritation, inflammation in the mouth and allergies resulting from keeping the pouches in the mouth.

Another advantageous attribute of nicotine pouches over other new forms of nicotine delivery is the likelihood of their low attractiveness to adolescents. Given their design characteristics, it is difficult to imagine that these products would become 
an attractive "gadget" for young people, as it was the case with e-cigarettes. The documented lack of interest in nicotine pouches by adolescents would be significant in stopping them from being perceived as a gateway for young people to tobacco products.

The authors accurately identify research gaps (e.g., pharmacokinetics of nicotine delivery in the presence of various flavorings, effect on smoking cessation) and propose a rational approach to nicotine pouches regulation. Early implementation of such regulations would help to proactively mitigate and potentially avoid problems faced by e-cigarette products despite being on the market for several years.

\section{References}

1. https://go77.pl/ (accessed 25 April 2021).

2. https://ec.europa.eu/health/sites/health/files/scientific_committees/scheer/docs/scheer_o_017.pdf (accessed 25 April 2021) 